\title{
Intrathecal Urokinase Infusion Through a Microcatheter into the Cisterna Magna to Prevent Cerebral Vasospasm: Experimental Study in Dogs
}

\author{
Takamasa Mizuno, Jun-ichiro Hamada, Yutaka Kai, Tatemi Todaka, \\ Motohiro Morioka, and Yukitaka Ushio
}

\begin{abstract}
BACKGROUND AND PURPOSE: Our preliminary report on intrathecal urokinase (UK) infusion into the cisterna magna $(\mathrm{CM})$ with a microcatheter showed good results in terms of vasospasm prevention in humans. In this study, we evaluated the relationship between different urokinase (UK) infusion sites and their effect on vasospasm prevention by using our canine subarachnoid hemorrhage (SAH) model.

METHODS: At 24 hours after SAH induction, we injected $1000 \mathrm{IU} / \mathrm{kg}$ UK into the cisterna magna (CM) or lumbar sac (LS) of dogs by using a microcatheter inserted at the lumbar region. We then obtained serial angiograms and chronologically examined the changes in the mean diameter of the basilar artery (BA) during a 14-day period to determine the effect of the different injection sites on vasospasm prevention. At 24 hours after UK injection, one dog from each group was killed for gross inspection of the subarachnoid clot. To measure its concentration in the CM and sylvian fissure, UK (1000 IU/kg) was injected into the CM or LS of dogs without SAH; measurements were taken at 15-minute intervals until 4 hours after injection.

RESULTS: At 24 hours after UK injection, subarachnoid clot in front of the brain stem persisted strongly in the LS group; it had almost disappeared in the CM group. In the LS group, there was severe and persistent BA constriction during the 14-day observation period. In the CM group, the BA was constricted on day 3; however, gradual dilatation occurred over time. The mean diameter of the BA on days 7, 10, and 14 was $48.2 \%, 53.9 \%$, and $58.9 \%$ in the LS group and $62.6 \%, 70.5 \%$, and $82.3 \%$ in the $\mathrm{CM}$ group. The difference between the two groups was significant on days 7,10 , and $14(P<.05)$. In dogs without $\mathrm{SAH}$, the average maximum UK concentration in the $\mathrm{CM}$ and the sylvian fissure was 2.5 and 6.7 times higher, respectively, in the CM group than in the LS group.

CONCLUSIONS: In our canine SAH model, the administration of UK into the CM was significantly more effective in preventing cerebral vasospasms than was administration into the LS.
\end{abstract}

Subarachnoid blood and/or its breakdown products induce chronic cerebral vasospasms in patients with subarachnoid hemorrhage (SAH) (1-5). When aneurysms are clipped by direct surgery, clots in the subarachnoid space can be eliminated and fibrinolytic agents can be delivered into the cistern via the cisternal drainage route (6-8). However, embolization of the aneurysm by endovascular surgery does not allow

Received August 6, 2002; accepted after revision November 7. From the Department of Neurosurgery, Kumamoto University School of Medicine, Japan.

Address reprint requests to Takamasa Mizuno, MD, Department of Neurosurgery, Kumamoto University School of Medicine, 1-1-1, Honjo, Kumamoto 860-8556, Japan.

(C) American Society of Neuroradiology removal of the subarachnoid clot or infusion of fibrinolytic agents directly into the cistern.

According to Kinugasa et al (9), endovascular embolization of aneurysms combined with intrathecal retrograde infusion of tissue plasminogen activator (t-PA) by means of spinal drainage can decrease the rate of symptomatic vasospasm. We reported an anterograde infusion method to achieve direct clot lysis after embolization of the ruptured aneurysm by delivering urokinase (UK) through a microcatheter inserted into the cisterna magna (CM) with use of the lumbar puncture maneuver (10). In our preliminary study, this resulted in the rapid clearance of subarachnoid hematomas and helped to prevent the occurrence of vasospasms in patients with ruptured aneurysms (10). In this study, to elucidate the relationship 
between the site of infusion of fibrinolytic agents and spasm prevention, we designed an experiment, by using our canine SAH model (11), to determine the effect of different injection sites on vasospasms. We also recorded the chronologic changes in the concentration of UK administered intrathecally from different sites.

\section{Methods}

All experiments were performed with the prior approval of the Animal Experimentation Ethics Committee of Kumamoto University and according to its Animal Care Guidelines.

\section{Experiment 1: Effect of UK Infusion Sites on Vasospasm Prevention}

Animal Groups._Adult mongrel dogs $(\mathrm{n}=18)$ weighing between 10 and $15 \mathrm{~kg}$ were used. At 24 hours after SAH induction, they were randomly divided into three groups and six animals in each group received an injection of physiologic saline into the ventral CM (control group), UK into the lumbar sac ([LS] LS group), or UK into the ventral CM (CM group). At 24 hours after injection, one dog from each group was killed for gross inspection of residual clots in front of the brain stem. In the remaining five dogs in each group, we obtained angiograms on days $3,7,10$, and 14 to evaluate chronologic changes in the basilar artery (BA) diameter.

Experimental SAH Model.-We detailed the procedures for cerebral angiography, microcatheter introduction into the ventral CM, and induction of SAH elsewhere (11). Briefly, all 18 dogs were anesthetized with intravenous pentobarbital, the left vertebral artery was catheterized up to the $\mathrm{C} 4$ spinal level, and baseline angiograms of the BA were obtained. A puncture was placed at the L2-3 interface, and a Tracker-18 microcatheter (Boston Scientific Corp., Boston, MA) was introduced into the ventral CM under fluoroscopic visualization. Autologous blood $(0.5 \mathrm{ml} / \mathrm{kg})$ was injected into the ventral $\mathrm{CM}$ through the microcatheter, and the head of the animal was lowered for 30 minutes to permit pooling and clotting of blood around the BA.

Intrathecal Infusion of UK.-At 24 hours after SAH induction, a microcatheter was reintroduced into the subarachnoid space by using the method employed for SAH induction. UK (1000 IU/kg; Mochida Pharmaceutical Co., Ltd., Tokyo, Japan), dissolved in $2 \mathrm{ml}$ of physiologic saline, was injected into the subarachnoid space $(1 \mathrm{ml} / \mathrm{min})$ through the microcatheter. The $1000 \mathrm{IU} / \mathrm{kg}$ volume of UK was deemed appropriate based on our preliminary clinical experience (10). The dogs were randomly assigned to one of three experimental groups consisting of six animals each. The control group received $2 \mathrm{ml}$ of physiologic saline delivered into the ventral CM by means of the microcatheter. The other dogs received an injection of UK solution into the LS (LS group) or ventral CM (CM group). In the LS group, the microcatheter was positioned at L2-3 for delivery of UK into the subarachnoid space.

Gross Observation of Residual Clots in Front of the Brain Stem.-At 24 hours after UK or physiologic saline injection, one dog from each group was killed with an intravenous bolus injection $(50 \mathrm{~mL} / \mathrm{kg})$ of sodium pentobarbital for gross observation of the residual clot around the BA.

Angiographic Estimation of the BA Diameter.-Repeat angiograms were obtained on days $3,7,10$, and 14 after SAH induction and used to evaluate chronologic changes in the BA diameter (11). The degree of vasospasm at the different time points was recorded as the percentage change from baseline values (12). The measurements were repeated three times by three investigators (J.H., Y.K., T.T.) blinded to the animal groups.

\section{Experiment 2: Measurements of UK Concentration in the} $C M$ and Sylvian Fissure

Adult mongrel dogs $(\mathrm{n}=10)$ weighing $10-15 \mathrm{~kg}$ were used. To evaluate its concentration in the CM and sylvian fissure of dogs without SAH, UK (1000 IU/ml dissolved in $2 \mathrm{ml}$ of physiologic saline) was injected $(1 \mathrm{ml} / \mathrm{min})$ into the ventral $\mathrm{CM}$ (CM group, $n=5$ ) or the LS (LS group, $n=5$ ) by means of the microcatheter introduced into the subarachnoid space. In the LS group, the microcatheter was positioned at L2-3 during UK injection and then immediately advanced into the ventral CM for periodic sampling of the CSF; $1.0 \mathrm{ml}$ of clear CSF was withdrawn at 15-minute intervals until 4 hours after injection. The first $0.6 \mathrm{ml}$ of CSF was discarded, and the next $0.4 \mathrm{ml}$ was immediately frozen $\left(-80^{\circ} \mathrm{C}\right)$ for later analysis.

To determine the UK concentration in the sylvian fissure, before UK injection, a cisternal drainage tube was inserted into the right sylvian fissure of all 10 dogs. A burr hole was drilled into the right temporal bone, the dura was incised (1-2 $\mathrm{mm}$ ), and a soft vinyl tube (length, $70 \mathrm{~mm}$; outer diameter, $1.5 \mathrm{~mm}$; inner diameter, $0.5 \mathrm{~mm}$ ) was inserted along the sylvian fissure. A small amount of fibrin glue was used to seal the dural hole around the tube and prevent CSF reflux. After UK injection, $0.5 \mathrm{ml}$ of clear CSF was withdrawn through this tube at 15minute intervals until 4 hours after injection. The first $0.2 \mathrm{ml}$ was again discarded, and the next $0.3 \mathrm{ml}$ was immediately frozen $\left(-80^{\circ} \mathrm{C}\right)$ for later analysis.

The UK concentration was determined according to the method of Morita et al (13). Peptide-methylcoumarin amide (MCA) is the synthetic substrate of UK; the UK concentration was determined by measuring the fluorescence of 7-amino4methylcoumarin (AMC) liberated by the hydrolysis of peptide-MCA. A 1-ml aliquot of the substrate solution $(50 \mathrm{mmol} / \mathrm{L}$ Tris- $\mathrm{HCl}$ buffer, $\mathrm{pH} 8.0$, containing $0.1 \mathrm{mmol} / \mathrm{L}$ peptide-MCA, $100 \mathrm{mmol} / \mathrm{L} \mathrm{NaCl}$, and $10 \mathrm{mmol} / \mathrm{L} \mathrm{CaCl}_{2}$ ) was preincubated at $37^{\circ} \mathrm{C}$ for 2.5 minutes, the UK solution was added, and the mixture allowed to react for 10 minutes at $37^{\circ} \mathrm{C}$. The reaction was terminated by adding $1.5 \mathrm{ml}$ of $17 \%$ acetic acid. The fluorescence of AMC was measured at an excitation wavelength of $380 \mathrm{~nm}$ and an emission wavelength of $460 \mathrm{~nm}$. The reaction was calibrated by using the UK standard product (1050 IU/vial) of the National Institute of Hygienic Sciences as a control.

\section{Statistical Analysis}

Data are expressed as the mean \pm standard error of the mean. Differences among the groups were determined by using the Student's $t$ test. Differences of $P<.05$ were considered to be statistically significant.

\section{Results}

\section{Experiment 1: Effect of UK Infusion Sites on Vasospasm Prevention}

In the LS and the control groups, there was strong persistence of residual clots around the BA at 24 hours after UK injection (Fig 1A). In the CM group, however, the clot had almost completely disappeared (Fig 1B). Representative serial angiograms of the BA are shown in Fig 2. The BA of dogs in the control and LS groups showed persistent narrowing on days 3, 7, 10, and 14 (Fig 2A, 2B). In the CM group, the BA was severely constricted on day 3 ; however, gradual dilatation occurred over the course of time (Fig 2C). Figure 3 is a graphic demonstration of the chronologic changes in the mean diameter of the $\mathrm{BA}$ in each group. In the control group $(\mathrm{n}=5)$, BA diameter was $55.8 \pm 12.8 \%$ of the pre-SAH value on day $3,49.0 \pm$ 

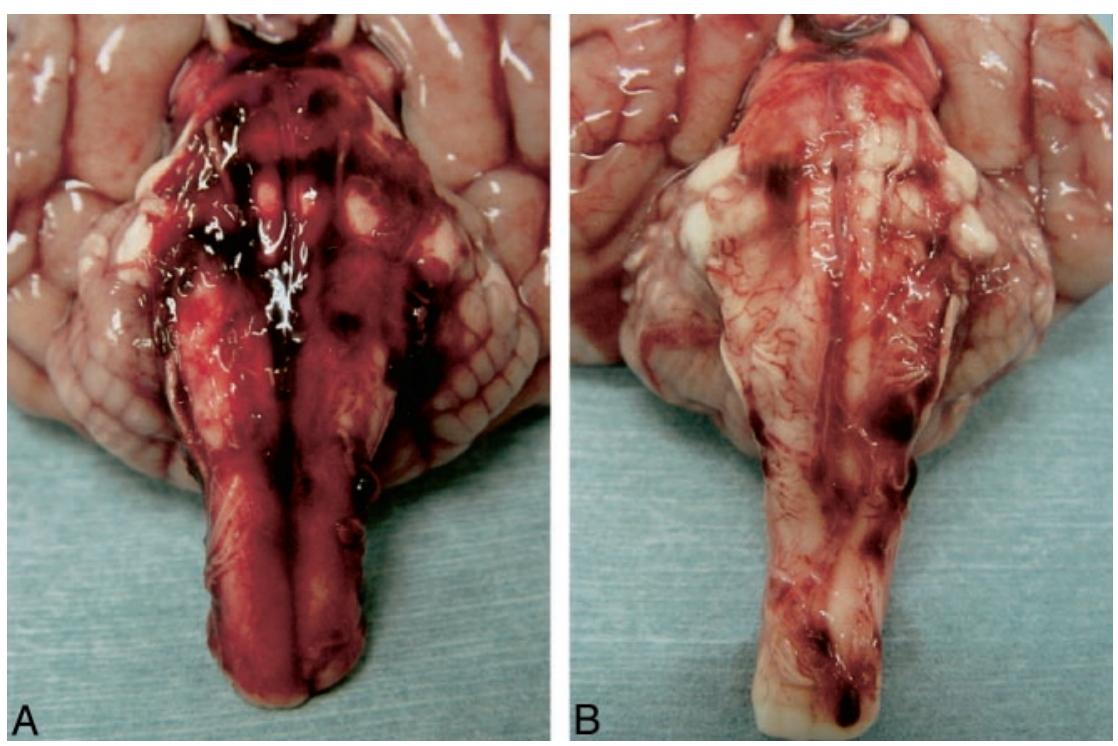

FIG 1. $A$ and $B$, Photographs of gross specimens show the ventral surface of the brain stem in dogs with experimental $\mathrm{SAH}$ at 24 hours after UK injection into the LS or CM. In the LS specimen $(A)$, there is strong persistence of the clot. However, in the CM specimen $(B)$, the clot around the brain stem has almost disappeared.

$2.8 \%$ on day $7,55.1 \pm 6.9 \%$ on day 10 , and $55.9 \pm$ $5.5 \%$ on day 14 . Severe constriction was noted on day 7; it persisted during the 14-day observation period. In the LS group $(\mathrm{n}=5)$, the BA diameter was $57.9 \pm$ $12.3 \%$ of the pre-SAH value on day $3,48.2 \pm 3.8 \%$ on day $7,53.9 \pm 6.4 \%$ on day 10 , and $58.9 \pm 7.7 \%$ on day 14. No significant arterial dilatation occurred during the 14-day observation period, and no significant differences were noted between the LS group and the control group. In the CM group $(\mathrm{n}=5)$, however, the BA diameter was $54.6 \pm 7.8 \%$ of the baseline on day $3,62.6 \pm 6.4 \%$ on day $7,70.5 \pm 9.3 \%$ on day 10 , and $82.3 \pm 4.9 \%$ on day 14 . As in the LS and the control groups, on day 3 the BA was severely constricted in the $\mathrm{CM}$ group. However, in the dogs in the $\mathrm{CM}$ group, the BA gradually dilated thereafter and the BA diameter recovered to approximately $80 \%$ of the pre-SAH value by day 14 . The difference between the $\mathrm{CM}$ group and the other groups was statistically significant $(P<.05)$ on days 7,10 , and 14 , indicating that the injection site played an essential role in the effectiveness of UK.

\section{Experiment 2: UK Concentration in the CM and Sylvian Fissure}

The UK concentration in the CM is shown in Fig 4. UK was detected in the CM until 4 hours after injection, regardless of the injection site (CM or LS). In the $\mathrm{CM}$ group $(\mathrm{n}=5)$, the average maximum UK concentration in the $\mathrm{CM}$ was $3557.5 \pm 270.8 \mathrm{IU} / \mathrm{ml}$. During the first 60 minutes after injection, it decreased rapidly; during the next 180 minutes, it declined gradually to $30 \mathrm{IU} / \mathrm{ml}$, the limit of detection. In the LS group $(\mathrm{n}=5)$, the UK concentration in the $\mathrm{CM}$ gradually increased during the first 60 minutes; the average maximum concentration was $1433.3 \pm$ $228.5 \mathrm{IU} / \mathrm{ml}$, less than $40 \%$ of that in the CM group. Then, as in the CM group, it gradually decreased. The average maximum UK concentration was about 2.5 times higher in the CM group than in the LS group.
Compared with the LS group, the concentration in the CM group was significantly higher at $15(P=$ $.0018)$ and $30(P=.0011)$ minutes after injection; there was no apparent difference between the two groups after 45 minutes.

The UK concentration at the sylvian fissure is shown in Fig 5. UK was detected at the sylvian fissure until 2 hours after injection regardless of the injection site (CM or LS). The average maximum UK concentration in the CM group $(\mathrm{n}=5)$ was $2066.5 \pm 718.6$ $\mathrm{IU} / \mathrm{ml}$ at 15 minutes; in the LS group $(\mathrm{n}=5)$ it was $309.2 \pm 66.0 \mathrm{IU} / \mathrm{ml}$ at 45 minutes. It was about 6.7 times higher in the CM group than in the LS group. Significant differences between the two groups were noted until 105 minutes after injection.

\section{Discussion}

\section{Experiment 1: Effect of UK Infusion Site on Vasospasm Prevention}

Our preliminary report (10) on intrathecal UK infusion into the $\mathrm{CM}$ by means of a microcatheter demonstrated good results in terms of vasospasm prevention. In all 15 patients with Hunt and Hess grades 3-4, CT scans obtained within 24 hours after the final UK infusion disclosed almost complete clearance of clots from the basal cistern and bilateral proximal sylvian fissures. Only one patient developed a transient neurologic deficit, and no patients manifested permanent delayed neurologic deficits as a result of vasospasm. This strongly suggests that the placement of the infusion tube in the CM effectively prevented the occurrence of symptomatic vasospasms.

In the current experimental study, we demonstrated that direct injection of UK into the CM was significantly more effective than injection into the LS for clot lysis and vasospasm prevention. The LS group manifested strong persistence of subarachnoid clots around the BA even at 24 hours after UK delivery, 

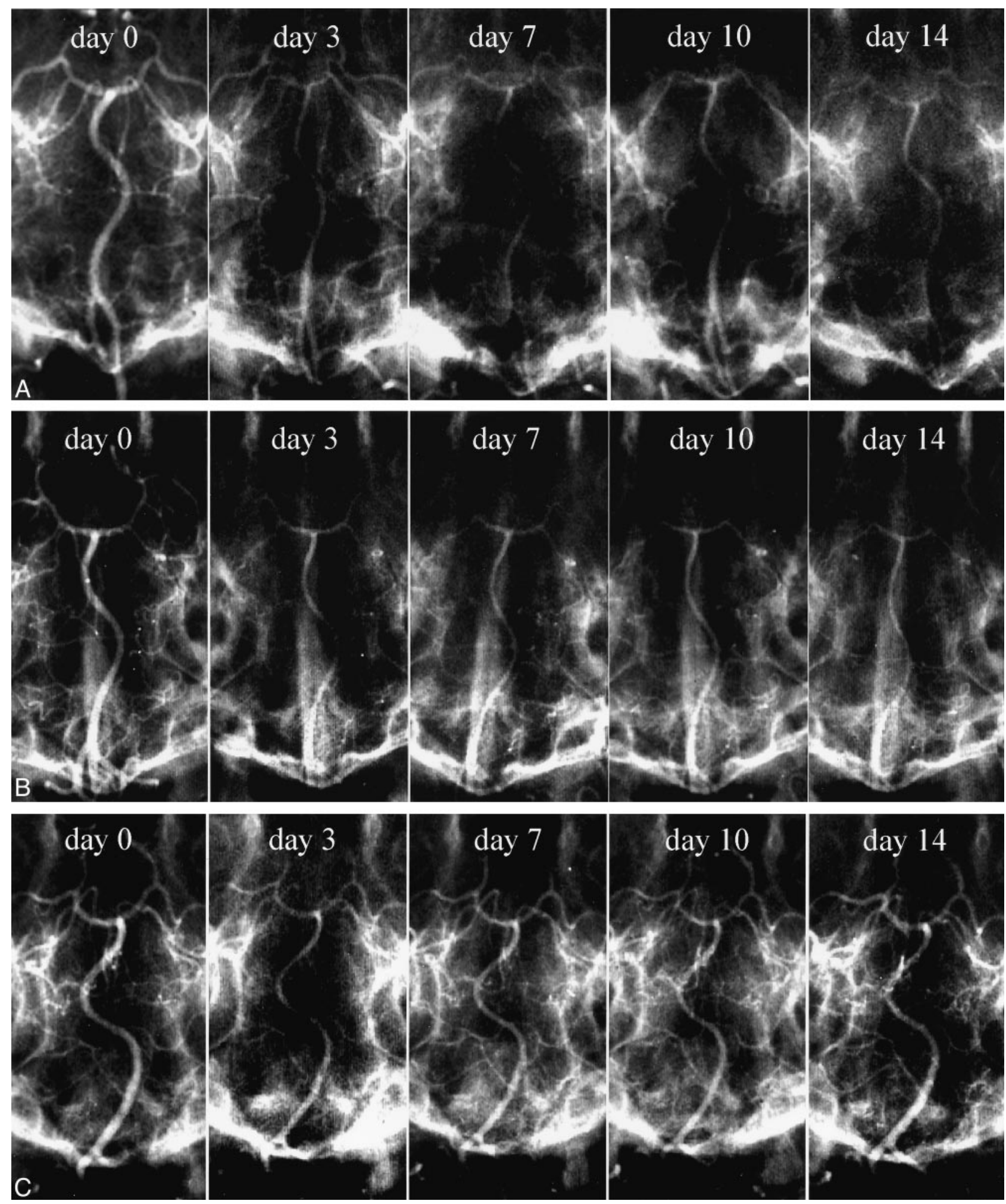

FIG 2. $A-C$, Representative serial angiograms of the canine BA in the control $(A), \mathrm{LS}(B)$, and CM $(C)$ groups. At 24 hours after SAH induction, UK (1000 IU/kg) or physiologic saline was delivered into the CM or LS. In the control group, physiologic saline was injected into the CM. The diameter of the BA was dilated on day 7 in the CM group compared with the other two groups. By day 14 , it almost returned to the pre-SAH size.

and there was no apparent dilatation effect on the BA during our 14-day observation period. In the CM group, however, subarachnoid clots had almost disappeared by 24 hours after UK injection and the BA became increasingly dilated on days 7,10 , and 14 . Our current results confirm our preliminary findings (10) that for successful intrathecal fibrinolytic therapy by spinal drainage it is important to advance the infusion 


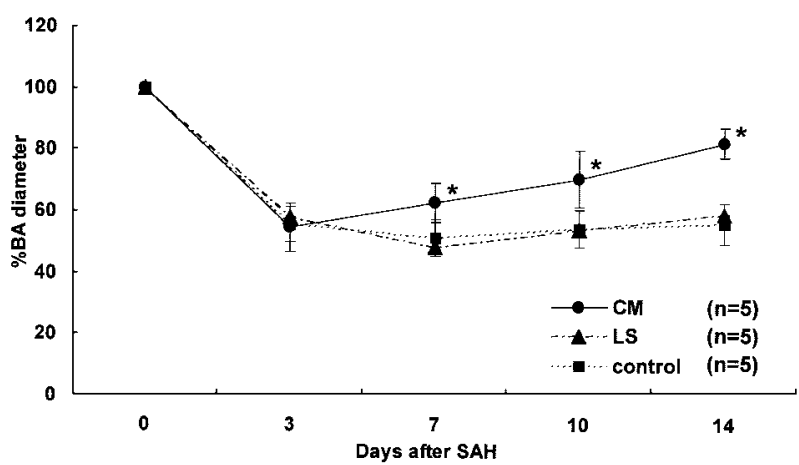

FIG 3. Graphic presentation of the percent changes in the mean diameter of the BA. The BA in the CM group was dilated on days 7,10 , and 14. No dilation was observed in the LS and control groups. There were significant differences between the CM group and the LS and control groups on days 7, 10, and 14 ( ${ }^{*}$ indicates $P<.05$ ).

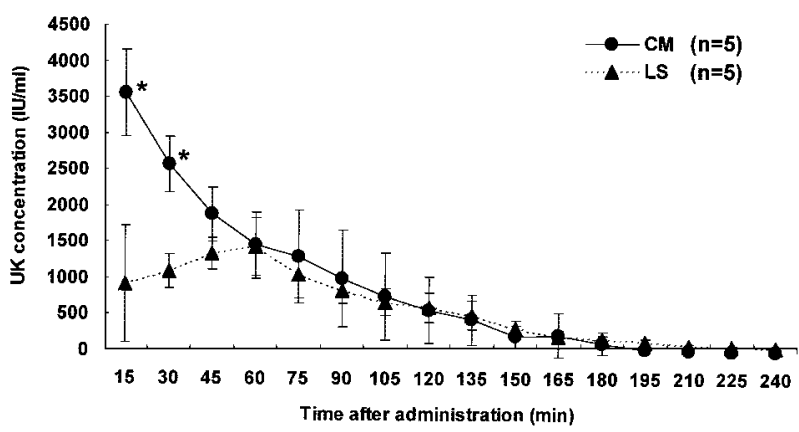

FIG 4. Time-course changes in the UK concentration in the $\mathrm{CM}$ after intrathecal injection. UK (1000 IU/ $/ \mathrm{kg})$ was injected into the CM or LS. Differences between the groups were significant until 30 minutes after injection (* indicates $P<.05$ ).

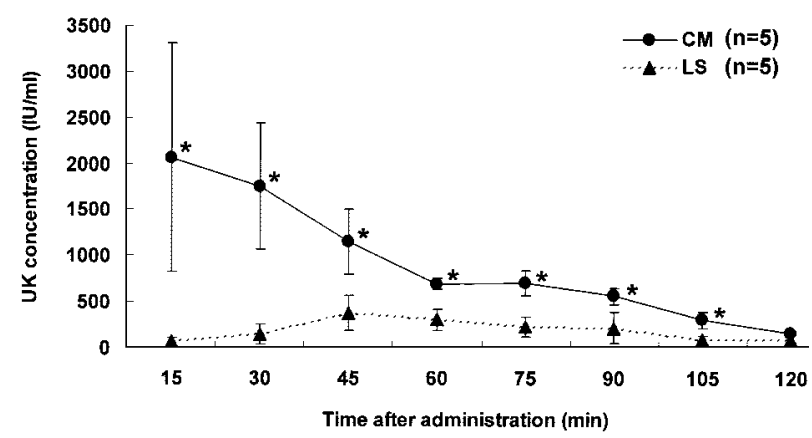

FIG 5. Time-course changes in the UK concentration at the sylvian fissure after intrathecal injection. UK (1000 IU/kg) was injected into the CM or LS. Differences between the groups were significant until 105 minutes after injection $\left({ }^{*}\right.$ indicates $P<.05$ ).

tube into the CM. Therefore, we conclude that UK infusion from the $\mathrm{CM}$ more effectively prevents cerebral vasospasm than does infusion from the lumbar subarachnoid space.

Although the subarachnoid clot had almost disappeared at 48 hours after SAH induction in the CM group, severe arterial constriction was present on day 3 . Findlay et al (14) suggest that the severity of vasospasm parallels the duration of contact between the blood clot and the cerebral vessels. This suggests that early UK injection may attenuate BA constriction in the acute phase after SAH. Other yet unknown mech- anisms may be involved whose induction is not prevented even by successful clot lysis. Further study is needed to gain a better understanding of this phenomenon.

\section{Experiment 2: UK Concentration in the CM and Sylvian Fissure}

Because the injection site played a significant role in the effectiveness of UK to prevent vasospasm, we determined how the delivery site affected the UK concentration in the $\mathrm{CM}$ of dogs without SAH. The average maximum UK concentration was 2.5 times higher in the CM than in the LS group (3557.5 \pm 270.8 vs $1433.3 \pm 228.5 \mathrm{IU} / \mathrm{ml}$ ). During the first 60 minutes, the area under the drug concentration-time curve was about two times larger in the CM group than in the LS group and the average maximum concentration in the LS group was only about $40 \%$ of that in the CM group. We attribute the superior spasm prevention effect of UK in the CM group to the difference in its $\mathrm{CM}$ concentration.

Two factors may account for the lower UK concentration in the CM after injection into the LS. First, the CSF temperature and $\mathrm{pH}$ may lead to a decrease in the enzymatic activity of UK as it moves from the LS to the CM. Second, in this model, UK may become diluted by diffusion throughout the subarachnoid space. As we noted only a slight concentration decrease when we incubated UK in artificial $\operatorname{CSF}\left(37^{\circ} \mathrm{C}\right.$, 4 hours, data not shown), we posit that the UK concentration decrease in the non-SAH LS group is due primarily to dilution.

The average maximum UK concentration in the LS group decreased to about $1400 \mathrm{IU} / \mathrm{ml}$, probably due to dilution. According to in vitro studies by Yoshida et al (15), this concentration was sufficiently high for clot lysis. However, we did not observe clot lysis or vasospasm prevention in our dogs in the LS group. We posit that this was partly because plasminogen activator inhibitor-1 (PAI-1) appeared in the CSF after SAH. This member of the acute-phase response proteins plays a role in the inhibition of fibrinolysis, is present in the CSF of patients with SAH (16), and may inactivate the enzymatic activity of UK. In our LS group, the presence of PAI-1 in the CSF may have led to a reduction in the activity of UK as it was transported from the LS to the CM, resulting in a much lower than expected UK concentration in the $\mathrm{CM}$. These considerations suggest that to overcome the UK concentration decrease by CSF dilution and the effect of PAI-1 inactivation, UK should be delivered directly into the CM to achieve an effective UK concentration in the subarachnoid space around the clot.

Until 105 minutes after the injection of $1000 \mathrm{IU} / \mathrm{kg}$, the UK concentration in the sylvian fissure was also significantly higher in the CM than in the LS group. The average maximum UK concentration at the sylvian fissure was about 6.7 times in the $\mathrm{CM}$ group than in the LS group. In the current study, we did not evaluate cerebral vasospasms of the anterior circula- 
tion. However, based on the observations reported here, we suspect that the lysis of clots in the sylvian fissure was more readily achieved by delivery of the fibrinolytic agent into the CM than the LS and posit that this accounts for the favorable results obtained in our preliminary study (10).

The optimal means for delivering fibrinolytic agents remains controversial. Multiple injections of small doses of t-PA result in the maintenance of higher levels of t-PA activity over longer periods than does the administration of a single injection and may be more effective in dissolving the hematoma $(9,17)$. Using our intrathecal UK induction method, we are continuing studies to determine the optimal UK delivery mode and concentration.

\section{Conclusion}

In our canine SAH model, the injection of UK into the $\mathrm{CM}$ resulted in significantly higher UK concentrations in the CM and in more effective clot lysis and reduction of cerebral vasospasms than did the administration of identical UK volumes into the LS. As effective clot lysis requires fairly high concentrations of UK around the clot, our method, which injects UK directly into the CM, is superior to previously reported methods.

\section{Acknowledgments}

We thank Mr. Kunihiko Takagi and Mr. Hideki Kiguchi for measuring the UK concentrations.

\section{References}

1. Fisher CM, Kistler JP, Davis JM. Relation of cerebral vasospasm to subarachnoid hemorrhage visualized by computerized tomographic scanning. Neurosurgery 1980;6:1-9

2. Kistler JP, Crowell RM, Davis KR, et al. The relation of cerebral vasospasm to the extent and location of subarachnoid blood visualized by CT scan: a prospective study. Neurology 1983;33:424-436

3. Espinosa F, Weir B, Overton T, Castor W, Grace M, Boisvert D. A randomized placebo-controlled double-blind trial of nimodipine after SAH in monkeys, I: clinical and radiological findings. $\mathrm{J} \mathrm{Neu}$ rosurg 1984;60:1167-1175

4. Seifert V, Eisert WG, Stolke D, Goetz C. Efficacy of single intracisternal bolus injection of recombinant tissue plasminogen activator to prevent delayed cerebral vasospasm after experimental subarachnoid hemorrhage. Neurosurgery 1989;25:590-598

5. Weir B. The pathophysiology of cerebral vasospasm. $\mathrm{Br}$ J Neurosurg 1995;9:375-390

6. Mizoi K, Yoshimoto T, Fujiwara S, Sugawara T, Takahashi A, Koshu K. Prevention of vasospasm by clot removal and intrathecal bolus injection of tissue-type plasminogen activator: preliminary report. Neurosurgery 1991;28:807-813

7. Findlay JM, Weir BKA, Kassell NF, Disney LB, Grace MGA. Intracisternal recombinant tissue plasminogen activator after aneurysmal subarachnoid hemorrhage. J Neurosurg 1991;75:181-188

8. Ohman J, Servo A, Heiskanen O. Effect of intrathecal fibrinolytic therapy on clot lysis and vasospasm in patients with aneurysmal subarachnoid hemorrhage. J Neurosurg 1991;75:197-201

9. Kinugasa K, Kamata I, Hirotsune N, et al. Early treatment of subarachnoid hemorrhage after preventing rerupture of an aneurysm. J Neurosurg 1995;83:34-41

10. Hamada J, Mizuno T, Kai Y, Morioka M, Ushio Y. Microcatheter intrathecal urokinase infusion into cisterna magna for prevention of cerebral vasospasm: preliminary report. Stroke 2000;31:2141-2148

11. Mizuno T, Hamada J, Kai Y, Todaka T, Morioka M, Ushio Y. Single blood injection into the ventral cisterna magna through a microcatheter for the production of delayed cerebral vasospasm: experimental study in dogs. AJNR Am J Neuroradiol 2003;24:608-612

12. Ohkuma H, Parney I, Megyesi J, Ghahary A, Findlay JM. Antisense preproendothelin-oligo DNA therapy for vasospasm in a canine model of subarachnoid hemorrhage. J Neurosurg 1999;90:1105-1114

13. Morita T, Kato H, Iwanaga S, Takada K, Kimura T. New fluorogenic substrates for alpha-thrombin, factor Xa, kallikreins, and urokinase. J Biochem (Tokyo) 1977;82:1495-1498

14. Findlay JM, Weir BKA, Kanamaru K, Grace M, Baughman R. The effect of timing of intrathecal fibrinolytic therapy on cerebral vasospasm in a primate model of subarachnoid hemorrhage. $\mathrm{Neu}$ rosurgery 1990;26:201-206

15. Yoshida Y, Ueki S, Takahashi A, Takagi H, Torigoe H, Kudo S. Intrathecal irrigation with urokinase in ruptured cerebral aneurysm cases: basic study and clinical application. Neurol Med Chir (Tokyo) 1985;25:989-997

16. Ikeda K, Asakura H, Futami K, Yamashita J. Coagulative and fibrinolytic activation in cerebrospinal fluid and plasma after subarachnoid hemorrhage. Neurosurgery 1997;41:344-349; discussion 349-350

17. Zabramski JM, Spetzler RF, Lee KS, et al. Phase I trial of tissue plasminogen activator for the prevention of vasospasm in patients with aneurysmal subarachnoid hemorrhage. $J$ Neurosurg 1991;75: 189-1961 